Supplement of Adv. Geosci., 45, 147-153, 2018

https://doi.org/10.5194/adgeo-45-147-2018-supplement

(C) Author(s) 2018. This work is distributed under

the Creative Commons Attribution 4.0 License.

(c) (1)

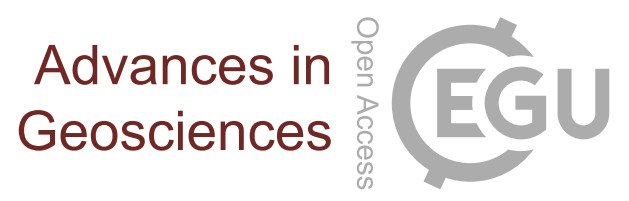

Supplement of

\title{
Large-scale assessment of Prophet for multi-step ahead forecasting of monthly streamflow
}

Hristos Tyralis and Georgia A. Papacharalampous

Correspondence to: Hristos Tyralis (montchrister@gmail.com)

The copyright of individual parts of the supplement might differ from the CC BY 4.0 License. 


\section{S.1 Definitions and background information}

Let us consider a time series of $N$ observations. Let us also consider a prediction made for the next $n$ observations.

If the total information utilized for this prediction is past available information (with respect to the prediction period), the prediction is also considered as a forecast.

For $n=1$, the forecast is considered as an one-step ahead forecast.

For $n>1$, the forecast is considered as a multi-step ahead forecast.

The forecasted variable(s) are called predictand (or dependent) variables and result via regression on the predictor (or independent) variables.

The latter can be endogenous or exogenous. According to Hyndman and Kostenko (2006) the former are variables, the values of which are determined within the system, and the latter are variables, the values of which are determined outside of the system.

Box S.1. Basic forecasting terminology.

Let $\{\underline{\mathrm{x}} \mathrm{t}\}, t=1,2, \ldots$ be a Hurst-Kolmogorov process. The latter is a three-parameter normal stationary stochastic process in discrete time. Its parameters $\mu, \sigma$ and $H$ are defined by Equations S.1-S.3 (Tyralis and Koutsoyiannis 2011).

$$
\begin{gathered}
\mu:=\mathrm{E}[\underline{\underline{X} t}] \\
\sigma:=(\operatorname{Var}[\underline{\underline{X}} t])^{1 / 2} \\
\rho_{k}:=\operatorname{Corr}[\underline{X} t, \mathrm{X} t+k]=|k+1|^{2 H} / 2+|k-1|^{2 H} / 2-|k|^{2 H}, k=0,1, \ldots, H \in(0,1)
\end{gathered}
$$

The parameter $\mu$ is the mean of the stochastic process and the parameter $\sigma$ is its standard deviation. The parameter $H$ represents the magnitude of long-range dependence, i.e. the tendency of wet or dry years to be clustered in long time periods (persistence), while the autocorrelation function $\rho_{k}$ increases with $H$. High values of $H$ denote strong long-term persistence, while when $H<0.5$, the resulting stochastic process is antipersistent, but still stationary. $H=0.5$ is equivalent to a stochastic process of independent variables.

Box S.2. Definition of the Hurst-Kolmogorov process. This box is an adapted reproduction from Tyralis et al. (2018).

The AutoRegressive Fractionally Integrated Moving Average (ARFIMA) models are widely used in hydrology. The reader is referred to Wei (2006, pp. 6-65, 489-494) for their detailed definitions. Let $d \in(-0.5,0.5)$. The stochastic process $\{\underline{x} t\}$ is an $\operatorname{ARFIMA}(p, d, q)$, if Equation S.4 holds.

$$
\varphi_{p}(\mathrm{~B})(1-\mathrm{B})^{d} \underline{x}_{t}=\theta_{q}(\mathrm{~B}) \underline{a}_{t}
$$


where

$$
\begin{gathered}
\varphi_{p}(\mathrm{~B}):=1-\varphi_{1} \mathrm{~B}-\ldots-\varphi_{p} \mathrm{~B}^{p} \\
\theta_{q}(\mathrm{~B}):=1+\theta_{1} \mathrm{~B}+\ldots+\theta_{q} \mathrm{~B}^{q} \\
\mathrm{~B}^{j} \underline{x}_{t}=\underline{X}_{t-j}
\end{gathered}
$$

B is an operator, $\varphi_{1}, \ldots, \varphi_{p}, d$ and $\theta_{1}, \ldots, \theta_{q}$ are parameters of the model, and $\left\{\underline{a}_{t}\right\}$ is a white noise process. The parameter $d$ is a measure of the estimated long-range dependence of the time series (see also Box S.2). The parameters $p$ and $q$ are the orders of the AutoRegressive and Moving Average components of the ARFIMA model. The ARFIMA forecasting method fits an $\operatorname{ARFIMA}(p, d, q)$ model to the fitting set. The fitting procedure is explained in Hyndman et al. (2017). The parameters $p$ and $q$ are estimated using the Hyndman and Khandakar (2008) algorithm and the parameter $d$ is estimated using the Haslett and Raftery (1989) algorithm. The method combines functions from the fracdiff (Fraley et al. 2012) and forecast (Hyndman et al. 2018) R packages. The fitted model is used for forecasting future values.

The Simple Exponential Smoothing (SES) model, introduced by Brown (1959) and described in Hyndman et al. (2008, p. 13), computes the forecast of the next period based on the forecast of the previous period, the latter adjusted using its error according to Equation S.8.

$$
f_{t+1}=f_{t}+a\left(x_{t}-f_{t}\right), a \in(0,1)
$$

The estimation of the parameters of the SES model is performed using procedures of the forecast R package (Hyndman et al. 2018).

The Prophet model, introduced by Taylor and Letham (2018) and implemented through the prophet $R$ package (Taylor and Letham 2017), considers time series forecasting as a curve-fitting exercise, while it does not explicitly consider the temporal dependence of the time series. It simultaneously uses a decomposable time series model. The Prophet model is inspired by the nature of the time series forecasted at Facebook, which are characterized by trend, multiple seasonality and holidays.

Box S.3. Brief description of the ARFIMA, SES and Prophet models. This box is an adapted reproduction from Papacharalampous et al. (2018c).

For the definitions we consider a time series of $N$ observations. Let us also consider a forecast of the last $n$ observations. Let $x_{1}, x_{2}, \ldots, x_{n}$ represent the last $n$ observations and $f_{1}$, $f_{2}, \ldots, f_{n}$ represent the forecasted values.

The Root Mean Square Error (RMSE) metric is defined by Equation S.9.

$$
\text { RMSE }:=\sqrt{(1 / n) \sum_{i=1}^{n}\left(f_{i}-x_{i}\right)^{2}}
$$

Let $\bar{x}$ be the mean of the observations, which is defined by Equation S.10. 


$$
\bar{x}:=(1 / n) \sum_{i=1}^{x_{i}}
$$

The Nash-Sutcliffe Efficiency (NSE) metric is defined by Equation S.11 (Nash and Sutcliffe 1970).

$$
\text { NSE }:=1-\left(\sum_{i=1}^{n}\left(f_{i}-x_{i}\right)^{2} / \sum_{i=1}^{n}\left(x_{i}-\bar{x}\right)^{2}\right)
$$

The index of agreement (d) metric is defined by Equation S.12 (Krause et al. 2005).

$$
d:=1-\left(\sum_{i=1}^{n}\left(f_{i}-x_{i}\right)^{2} / \sum_{i=1}^{n}\left(\left|f_{i}-\bar{x}\right|+\left|x_{i}-\bar{x}\right|\right)^{2}\right)
$$

Let $S_{x}$ be the standard deviation of the observations, which is defined by Equation S.13.

$$
s_{X}:=\sqrt{(1 /(n-1)) \sum_{i=1}^{n}\left(x_{i}-\bar{x}\right)^{2}}
$$

Let $\bar{f}$ be the mean of the forecasts and $s_{f}$ be the standard deviation of the forecasts, which are defined by Equations S.14 and S.15 respectively.

$$
\begin{gathered}
\bar{f}:=(1 / n) \sum_{i=1}^{n} f_{i} \\
S_{f}:=\sqrt{(1 /(n-1)) \sum_{i=1}^{n}\left(f_{i}-\bar{f}\right)^{2}}
\end{gathered}
$$

The Pearson's correlation coefficient (Pr) metric is defined by Equation S.16 (Krause et al. 2005).

$$
\operatorname{Pr}:=\left(\sum_{i=1}^{n}\left(x_{i}-\bar{x}\right)\left(f_{i}-\bar{f}\right)\right) /\left(\sum_{i=1}^{n}\left(x_{i}-\bar{x}\right)^{2} \sum_{i=1}^{n}\left(f_{i}-\bar{f}\right)^{2}\right)^{0.5}
$$

The Kling-Gupta Efficiency (KGE) metric is defined by Equation S.17 (Gupta et al. 2009).

$$
\mathrm{KGE}:=1-\sqrt{(\operatorname{Pr}-1)^{2}+\left(\left(s_{f} / s_{x}\right)-1\right)^{2}+((\bar{f} / \bar{x})-1)^{2}}
$$

Box S.4. Definition of the RMSE, NSE, $d$ and KGE metrics. This box is an adapted reproduction from Papacharalampous et al. (2018a, supplementary material). 


\section{S.2 Additional figures}

(a)

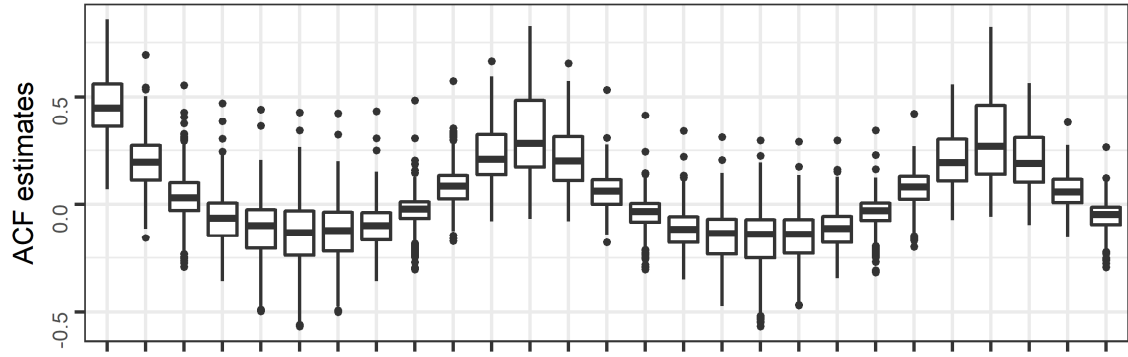

(b)

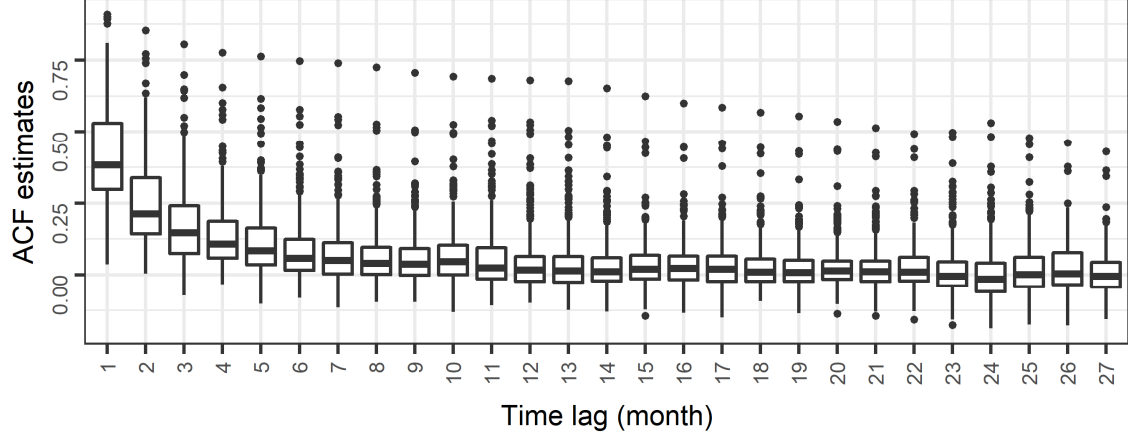

(c)

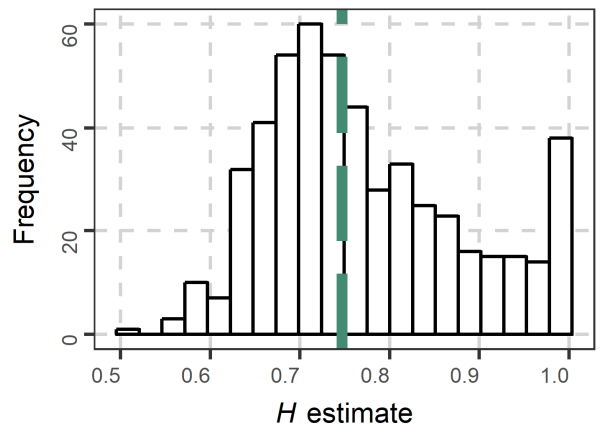

Figure S.1. Exploration of the monthly streamflow data examined in the forecasting experiment: (a) AutoCorrelation (ACF) estimates of the original time series, (b) ACF estimates of the deseasonalized time series and (c) Hurst parameter $(H)$ estimates of the deseasonalized time series; their median is denoted with a green dashed line. The ACF and $H$ estimates for the 513 catchments are presented in an aggregated form.
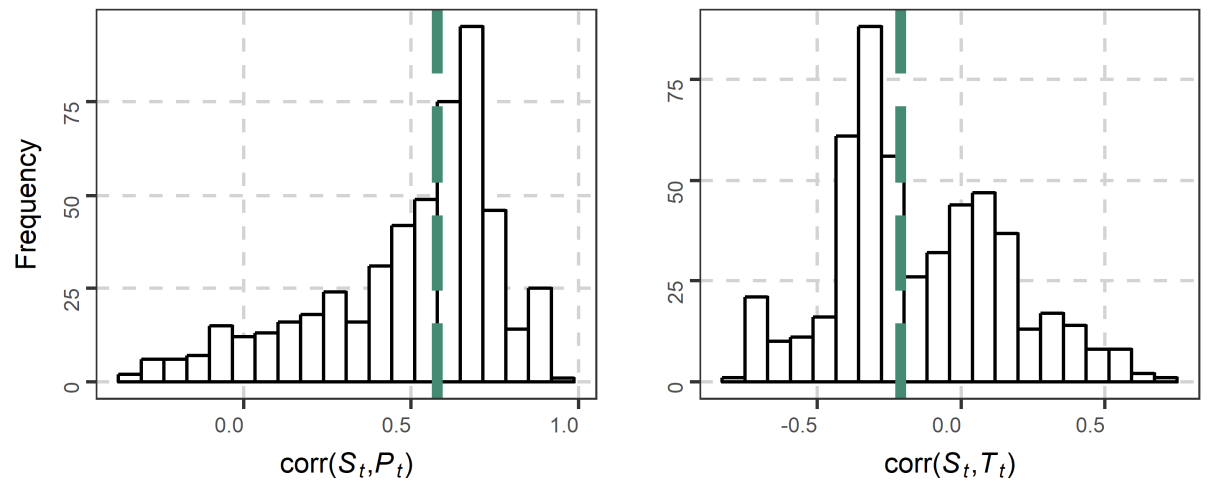

Figure S.2. Pearson's correlations between the monthly streamflow and precipitation variables $\left(\operatorname{corr}\left(S_{t}, P_{t}\right)\right)$, and the monthly streamflow and temperature variables ( $\left.\operatorname{corr}\left(S_{t}, T_{t}\right)\right)$ computed for the 513 catchments examined in the forecasting experiment. The correlations are presented in an aggregated form. Their medians are denoted with green dashed lines. 

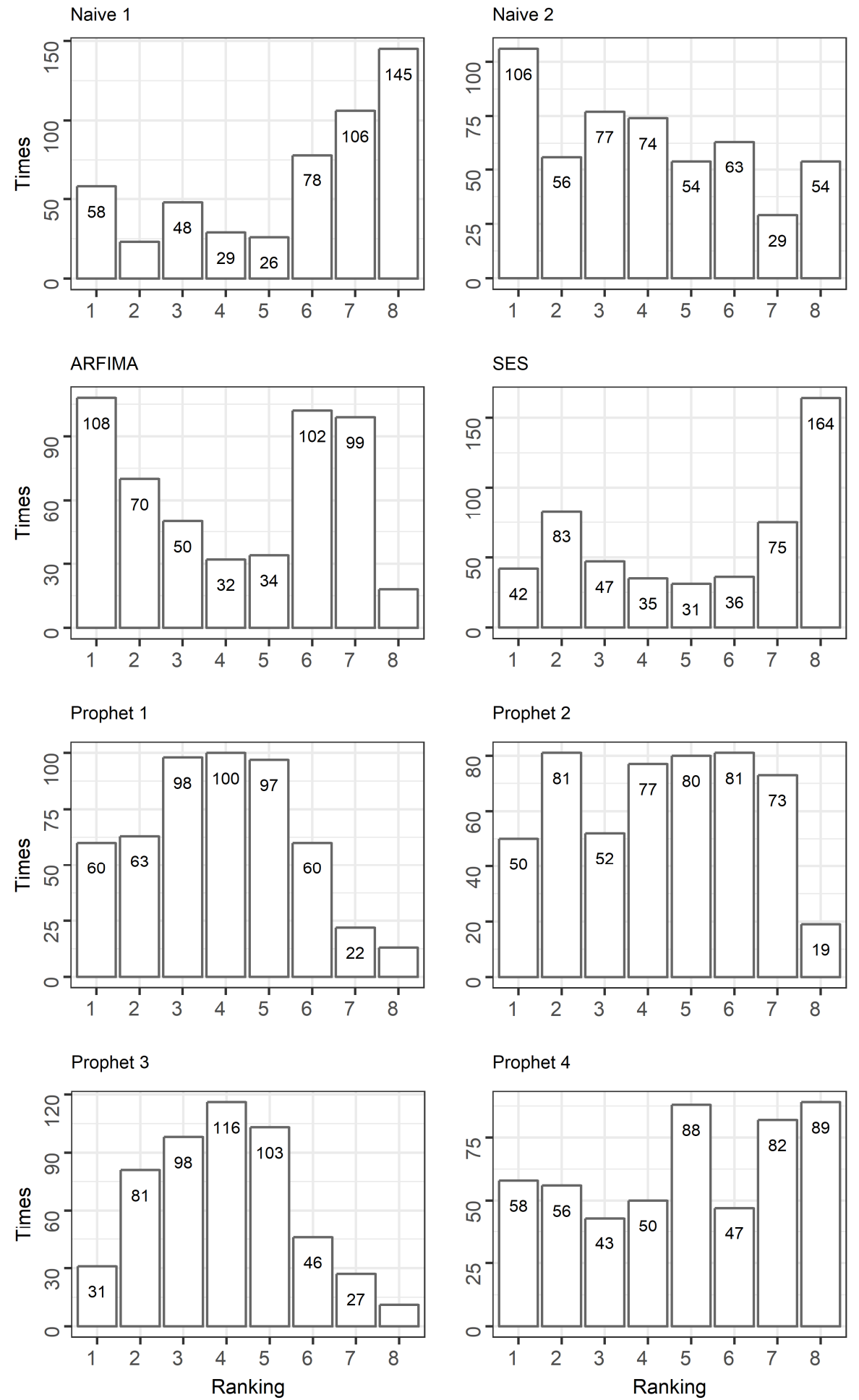

Figure S.3. Number of times that each algorithm is ranked from best ( $\left.1^{\text {st }}\right)$ to worst $\left(8^{\text {th }}\right)$ according to the RMSE metric. 


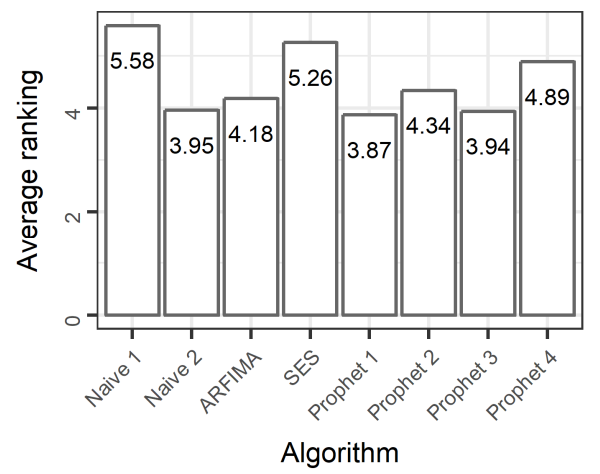

Figure S.4. Average rankings of the algorithms according to the RMSE metric. The algorithms are ranked from best $\left(1^{\text {st }}\right)$ to worst $\left(8^{\text {th }}\right)$.

\section{References (not included in the paper)}

Brown RG (1959) Statistical forecasting for inventory control. McGraw-Hill, New York Gupta HV, Kling H, Yilmaz KK, Martinez GF (2009) Decomposition of the mean squared error and NSE performance criteria: Implications for improving hydrological modelling. Journal of Hydrology 377(1-2):80-91. https://doi.org/10.1016/j.jhydrol.2009.08.003

Haslett J, Raftery AE (1989) Space-Time Modelling with Long-Memory Dependence: Assessing Ireland's Wind Power Resource. J R Stat Soc C-Appl 38(1):1-50. https://doi.org/10.2307/2347679

Hyndman RJ, Kostenko A (2006) Glossary of forecasting terms. https://robjhyndman.com/mwh3/FG4.pdf

Hyndman RJ, Koehler AB, Ord JK, Snyder RD (2008) Forecasting with exponential smoothing: The state space approach. Springer-Verlag Berlin Heidelberg, pp 3-7. https://doi.org/10.1007/978-3-540-71918-2

Krause P, Boyle DP, Bäse F (2005) Comparison of different efficiency criteria for hydrological model assessment. Advances in Geosciences 5:89-97

Nash JE, Sutcliffe JV (1970) River flow forecasting through conceptual models part I-A discussion of principles. Journal of Hydrology 10(3):282-290. https://doi.org/10.1016/0022-1694(70)90255-6

Wei WWS (2006) Time Series Analysis, Univariate and Multivariate Methods, second edition. Pearson Addison Wesley 